\title{
Assessment of the Primary Stability for implants inserted by CBCT-Guided Stereolithographic Templates using a Partially limiting design versus Completely limiting designs in the posterior mandible: Clinical Study
}

\author{
Khaled Taymour Khourshid*, Mohamed Katamish** , Moustafa \\ Mohamed Sayed Taha***
}

\section{Abstract :}

Implants have a higher success rate to support the prosthetic appliance when certain conditions are met during surgical procedure prompting to osseointegration. The stability that implant has gained at the placement time indicates the integrity of future osseointegration, and later predictable implant survival. The first requirement for implant placement is essential implant stability, which is guaranteed by the new bone arrangement and rebuilded around the implant in the repairing period. The implant has to remain stable to secure undisturbed bone development onto the implant surface

\section{Introduction:}

Nowadays, the implant-supported prosthesis are one of the highly recommended treatment options for management of completely or partially edentulous patients. Classically, a conventional mucoperiosteal flap was utilized to expose the bone, although recent efforts have been made to reduce the surgical discomfort to the patient. A minimally invasive techniques have been developed to provide the maximum functional and esthetical demands of the patient. fapless technique, as an

* BDS Misr international University

** Professor of oral and maxillofacial surgery Ain shams university

*** Associate Professor of oral and maxillofacial surgery Ain shams university 
example, has been preferred by many clinicians comparatively to the conventional surgical procedures, a fapless surgical approach was clearly advantageous in regard to preservation and protection to the bone*

\section{Review of literature:}

Available bone has special importance in implant placement, outlining the external architecture and volume of the edentulous area considered for implants to aid treatment planning. Edentulous posterior mandible follow specific pattern of bone loss, mainly in a vertical direction pattern that differs from other sites in the oral cavity which could be in both direction, vertical and horizontal. Classification for the different types of defect that could affect the posterior mandible has been established for better, reliable and more convenient way to communicate between surgeon all around the world(4).

The predictability of the outcome of an implant restoration in the posterior part of the mouth is dependent on many variables including but not exclusive to the following: Available space, Implant number and position, Occlusal considerations, Type of prosthesis, Overall treatment plan (5)dental implants/ treatment planning and posterior maxilla, and dental implants/treatment planning and posterior mandible. No exclusion criteria were used for the initial search. Clinical trials, randomized and non randomized studies, classical and comparative studies, multicenter studies, in vitro and in vivo studies, case reports, longitudinal studies and reviews of the literature were included in this review. RESULTS: One hundred and fifty-two articles

* esthetics, and comfort with a minimally invasive surgical approach. Flapless implant surgery has been proposed to fulfill these requirements. Traditionally, flapless implant surgery was carried out by using a tissue punch technique, which may be potentially harmful because of the inherent blindness of the technique. The purpose of this article is to introduce a predictable flapless approach for treatment of 2 patients through principles of computer-guided implantology. Materials and Methods: Using dedicated interactive computer software programs and 3D radiographic techniques such as computed tomography (CT). met the inclusion criteria of treatment planning of dental implants in posterior jaw and were read in their entirety. The selected articles were categorized with respect to their context on space for restoration, anatomic considerations (bone quantity and density.

Sufficient space must exist to allow the restorative dentist to fabricate restorations which are harmonious aesthetically with the adjacent teeth. On examination the space between the residual ridge and the opposing occlusal plane should be evaluated. Replacing premolar and molar teeth requires $10 \mathrm{~mm}$ of space between the residual ridge and the opposing occlusion, while $7 \mathrm{~mm}$ would be considered the minimum amount of space allowed. When teeth are missing for prolonged periods of time, opposing teeth overerupt and jeopardies the restorative space. If this is minimal, enameloplasty or minimal restorative therapy may be required to create space(5)dental implants/treatment planning and posterior maxilla, and dental implants/ treatment planning and posterior mandible. No exclusion criteria were used for the initial search. Clinical trials, randomized and non randomized studies, classical and comparative studies, multicenter studies, in vitro and in vivo studies, case reports, longitudinal studies and reviews of the literature were included in this review. RESULTS: One hundred and fifty-two articles met the inclusion criteria of treatment planning of dental implants in posterior jaw and were read in their entirety. The selected articles were categorized with respect to their context on space for restoration, anatomic considerations (bone quantity and density .

The rehabilitation with implant offers a lot of benefits when compared with other treatment option like a removable partial dentures, it offers better occlusion and support, simplification of the prosthesis, less invasive restorative procedures, maintain the bone and improvement in oral health(5)dental implants/ treatment planning and posterior maxilla, and dental implants/treatment planning and posterior mandible. No exclusion criteria were used for the initial search. Clinical trials, randomized and non randomized studies, 
classical and comparative studies, multicenter studies, in vitro and in vivo studies, case reports, longitudinal studies and reviews of the literature were included in this review. RESULTS: One hundred and fifty-two articles met the inclusion criteria of treatment planning of dental implants in posterior jaw and were read in their entirety. The selected articles were categorized with respect to their context on space for restoration, anatomic considerations (bone quantity and density. Excellent results in rehabilitation with dental implants necessitate meticulous attention to fine details especially the posterior quadrants of the mouth which offer challenge for rehabilitation with dental implants due to their anatomical and occlusal complexity (6).. The resorption of the alveolar ridge in the posterior quadrant, the presence of the inferior alveolar nerve, the quality of the bone is poor and the enormous occlusal forces formed lead to clinical environment that may threats the long-term biological and biomechanical success for the implant restoration(6).

The construction of the surgical guide Stereolithographic templates is created upon the following design concepts; Non-limiting design, Partially limiting design and Completely limiting design These design concepts are categorized based on the amount of surgical limitation existing by the surgical guide templates(7)(8)(9).

The Non-limiting design offers only an indication to the site, without any restraint to the surgeon as to where the proposed prosthesis should be paced in relation to the selected implant site. It only denotes the ultimate site of the implants without any regards for the angulation during drilling, therefore accepting abundant flexibility in the final sitting of the implant. (10)

In the partially limiting design, the concept aid the surgeon to precisely drill the osteotomy. It restrict the drill movement in all directions during the whole drilling process which include the pilot drill and all the subsequent dills, the angulation of the drill is also secured while drilling. The length of the osteotomy will be limited by the guide in order not to drill too much or too little and maintain the ideal length for the implant. The only step that could not be not offered by the partially limiting design is the implant placement step, in which the implant is placed by freehand. (7)(11)

In the Completely limiting design restrictions to all the instruments used to prepare the osteotomy in a buccolingual and mesiodistal direction. Furthermore, the addition of drill stops restrict the depth of the preparation, and thus, the positioning of the future prosthetic plane of the implant $(12)^{\prime \prime}$ where only osteotomy sites are prepared using sequential, removable surgical drilling guides (generated using computer software and through the process of stereolithography. Two popular designs exists : cast-based guided surgical guide and computer-assisted design, the manufacturing (CAD/CAM) based surgical guide(8).

The CAD/CAM-guided implant surgery could also be three dimensionally printed, and allows for construction of an immediately loaded prosthesis for a partially edentulous patient(13) for proper implant rehabilitation. The CT scanned images are transformed into data that are known by a CT imaging and planning software. The accuracy of CAD/ CAM technology in dental implant planning and expected transferal of the presurgical plan to the surgical site has been documented to be successful (14)(15)(16). The software then secures this presurgical plan to the surgical site by using stereolithographic template guides(17). Incorporation of prosthetic planning using a stereolithographic template permits the treatment to be enhanced from both point of views, prosthodontics and biomechanical (18)

There are three types of surgical guide that could be fabricated depending on the type of support they get: Bone, Mucosa, and Tooth supported. The Bone supported guides are mainly needed in case of partially and completely edentulous sites. If used in case of partially edentulous sites, a minimum of $3 \mathrm{~cm}$ of supporting bone must be present or missing three teeth that need restoration. Bone guides are needed in the presence of thin alveolar 
ridge. Furthermore the Mucosa supported guides are used in fully edentulous sites. The Advantage in that case is there will be less or no tissue reflection required (flapless), and so minimize the post-operative symptoms. Toothsupported guides on the other hand require at least three stable sound teeth to support the guide during the surgical procedure. (7)(19) (20)(17)

\section{Aim of the study :}

To assess primary stability of implants inserted by CBCT-guided stereolithographic templates using a partially limiting vs completely limiting design in posterior mandible.

\section{Methodology:}

The study was conducted on patients seeking rehabilitation in bilateral edentulous posterior area of the mandible. Patients were recruited from those attending the Oral and Maxillofacial Surgery Department, Faculty of Dentistry, Ain Shams University. Presurgical evaluation of all patient including clinical examination, $\mathrm{CBCT}$, and diagnostic cast were done. The surgical and radiographic protocol of the study were reviewed by the Faculty of Dentistry, Ain Shams University.

Before conduction of this study, the research protocol was reviewed and approved by the Research Ethical Committee of Faculty of Dentistry, Ain-Shams University. The patients were informed about the aim and design of the study, and written consents were obtained.

\section{Patient Selection and Grouping:}

\section{Inclusion criteria:}

Male and female patients with minimum age 18 years old, who are able to understand and sign an informed consent.

Bilateral edentulous posterior mandible

Physical status (ASA I or II according to American Society of Anesthesiologist )t

Interach space between residual ridge and opposing occlusion should be at least $7 \mathrm{~mm}$

The residual ridge should have at least 6 $\mathrm{mm}$ in diameter buccolingally .

The mesiodistal diameter of the residual ridge must allow for safe distance of 1.5 $\mathrm{mm}$ between the neighboring teeth and the implant, and $3 \mathrm{~mm}$ between two implants adjacent to each other.

Keratinized mucosa must be present (to secure at least $2 \mathrm{~mm}$ keratinized band around the implant)

\section{Exclusion criteria:}

Lingual concavity in the edentulous molar area of the mandible (68\% incidence) to avoid bleeding and infection in the parapharyngeal space

Approximation to the inferior alveolar canal (at least $2 \mathrm{~mm}$ must be secured for safe distance between the apical part and the margin of the canal)

Approximation to the mental foramen

Presence of any soft tissue or bony pathosis related to the posterior mandible

Patients with any parafunctional habit

Medically compromised patient;

Uncontrolled diabetes

Uncontrolled hypertensive

Treated or under treatment of I.V amino bisphosphonates

Subjected to irradiation in the head and neck area

Osteoradionecrosis

Sjogren's syndrome

Systemic lupus erythematosus

Osteoporosis

\section{Grouping;}

After the patients are proven to be eligible for this study, total of 14 implants were used. Each patient received at least two implants placed on each side (i.e. four implants per patient) in a split mouth design . The right side of the mandible received the implants using partially limiting design (universal kit) as group 
A, while the left side of the mandible received implants using the completely limiting design (specific kit) as group B. (Fig.1)

\section{Group A}

Using partially limiting (21) stereolithographic surgical guide (universal kit) in which only the drilling will be done through the metal sleeve in the guide, while the implant were placed hand free The right side of the mandible received the implants placed with the universal kit .

\section{Group B}

Usingcompletelylimitingstereolithographic surgical guide (specific kit), in which both the drillings and implant placement were be done through the metal sleeves in the surgical guide. The left side of the mandible received the implant placed with the specific kit.

Fabrication of stents

Impression were taken for construction of study cast

Waxing up for the study cast was made

CBCT for the study cast was made to register the soft tissue of the residual ridge.

$\mathrm{CBCT}^{*}$ for the study cast with the wax-up was made to register the position of the future prothesis

CBCT is obtained for the patient to provide information regarding dimensions of the hard tissue of the ridge

Both CBCT data are inputted into the planning software (In2Guide) ${ }^{* *}$

The software matches numerous points on the surface of the cast to the corresponding anatomical surface points in the CT data

Superimposition of both CBCTs to configure three dimension picture of the mandible and register the soft tissue

Mapping between the image data and the CBCT allows for the production of an accurate

\footnotetext{
* CBCT Machine, Planmeca Proface (Planmeca Oy Inc. Helsinki, Finland )

** CBCT Software, In2Guid3 Module of Ondemand 3D (Cybermed In. Seoul, Korea)
}

surgical guide

The surgical template is $3 \mathrm{D}$ printed ${ }^{* * *}$ later

Metal sleeves will be added for each implant site in the guide corresponding to the implant manufacture

The procedure.

Surgical Technique:

A crestal incision was made using a lancet blade no 15 (Fig 11)

Reflection of just the crestal bone using a muco-periosteal elevator

Traction suture were made in the buccal and lingual sides to prevent interference of the flap with seating of the guide.(Fig 12)

The surgical guide was seated on the mandibular arch and fixed to its position by anchoring pins in the molar area bilaterally

In the partially limiting design, metal sleeve for the universal kit were used that fit tightly into the surgical guide in the proposed implant site in the guide

Surgical kit for the Universal kit is used that correspond to the metal sleeve

Drilling was made sequentially according to the manufacture's guide. A drill guide was inserted before each drill corresponding to its size.

After the osteotomy is made (Fig ), removal of the surgical guide is required in the partially limiting design

The partially limiting design require removal of the guide completely and the implant will be inserted free hand (Fig 22

After insertion of the implant covering screw were added to each implant

In completely limiting side using specific kit, different type of metal sleeve were used corresponding to the kit.

In the completely limiting design also required specific surgical kit

Drilling was made sequentially according

*** 3 D Printer, Envision Tech (Enivision TEC Inc, Dearbon, Michigan, U.S) 
to the manufacture's guide. A drill guide was inserted before each drill corresponding to its size.

In the completely limiting design removal of the guide for insertion of the implant is not required (Fig) ;

as the implant can be inserted with the aid of Internal Implant Driver (Fig )

while the guide is fixed to mandible

and the Depth Stops (Fig ) engages the implant driver to place the at the proper depth through the metal sleeve

After all implants are inserted and prior to placing the covering screw, smart peg (Fig) would be applied to each implant to measure the primary stability (Fig)

Finally closure of the wound is secure by a resorbable suture 3.0 (Fig )

\section{Measuring the primary stability.}

The primary stability were measured using the Osstell ${ }^{\circledR} I S Q$ (Fig 26), which is a resonance frequency analysis device used to measure the implant stability quotient immediately after implant placement.

After the osteotomy preparation and immediately after implant placement, the smart peg (respective to the implant system) is attached to implant with help of the smart peg mount.

The mount is removed after securing the smart peg in the implant

The RFA(resonance frequency analysis) device is activated

The probe was maintained at(Fig 27,28);

$1-3 \mathrm{~mm}$ away from the smart peg

at 90 degrees' angle

$3 \mathrm{~mm}$ above soft tissue

Values are expressed as numbers between 1 and 100 in ISQ

Readings were taken in all directions, mesial, distally and buccally, and lingually since bone is not uniform all around the implant
The average of the two records is recorded as the ISQ value

\section{RESULTS}

This study has been conducted on 14 implants divided among patients according to their need. Patients were selected from those attending the implant clinic affiliated to Oral and Maxillofacial department, Faculty of Dentistry, Ain Shams University. Selection of patients was done fulfilling the predetermined inclusion and exclusion criteria. Implants were divided into two groups, Group A, which involves the right side of the mandible, the implants were inserted using partially limiting design (Universal kit ). Group B involves the left side of the mandible, implants were inserted using the completely limiting design ( specific kit). One females and two males, with age ranges from 20-60 years were involved in the study.14 implants were used in total, in which 7 implants were inserted by each kit at each side and all implants size were $3.8 * 9$.

The cases were evaluated clinically through the measurement of primary stability and radiographic accuracy .

The mean of the immediate postoperative osstel readings among Group A was 66.39 ISQ \pm 10.6.ISQ The mean of the immediate postoperative osstel readings among Group B was 68.25 ISQ \pm 9.91 ISQ. (Table 1,2)

Comparing the mean of osstel reading for primary stability between Group A and Group B reveled that there was no statistical difference between the two groups.

\section{Reference}

1. Rodrigo D, Rodrigo D, Aracil L, Martin C. Diagnosis of implant stability and its impact on implant survival : a prospective case series study. 2009;255-61.

2. Azari A, Nikzad S. Flapless Implant Surgery: Review of the Literature and Report of 2 Cases With Computer-Guided Surgical Approach. J Oral Maxillofac Surg. 2008;66(5):1015-21. 
3. Misch. This ebook is uploaded by dentalebooks . com This ebook is uploaded by dentalebooks . com. 2008. 206 p.

4. Cawood JI, Howell RA. A classification of the edentulous jaws Trauma; Preprosthetic Surgery. Int J Oral Maxillofac Surg. 1988;17(13):232-6.

5. Monteiro DR. Posterior partially edentulous jaws, planning a rehabilitation with dental implants. World J Clin Cases. 2015;3(1):65.

6. Blanes RJ, Bernard JP, Blanes ZM, Belser UC. A 10-year prospective study of ITI dental implants placed in the posterior region. I: Clinical and radiographic results. Clin Oral Implants Res. 2007;18(6):699-706.

7. Stumpel L. Cast-based guided implant placement: A novel technique. J Prosthet Dent. 2008 Aug 1;100:61-9.

8. D'Souza KM, Aras MA. Types of Implant Surgical Guides in Dentistry: A Review. J Oral Implantol. 2011;38(5):643-52.

9. Testori T, Robiony M, Parenti A, Luongo G, Rosenfeld AL, Ganz SD, et al. Evaluation of accuracy and precision of a new guided surgery system: a multicenter clinical study. Int J Periodontics Restorative Dent. 2014;34 Suppl 3(November):s59-69.

10. Spector L. Computer-Aided Dental Implant Planning. Dent Clin North Am. 2008;52(4):761-75.

11. Joda AT, Buser PD. Digital implant dentistry - a workflow in five steps. 2013;1620.

12. Mandelaris GA, Rosenfeld AL, King SD, Nevins ML. Computer-guided implant dentistry for precise implant placement: combining specialized stereolithographically generated drilling guides and surgical implant instrumentation. Int J Periodontics Restorative Dent. 2010;30(3):275-81.

13. Marchack CB. CAD/CAM-guided implant surgery and fabrication of an immediately loaded prosthesis for a partially edentulous patient. J Prosthet Dent. 2007;97(6):389-94.
14. Jabero M, Sarment DP. Advanced Surgical Guidance Technology: A Review. Implant Dent. 2006;15(2).

15. Valente F, Schiroli G, Sbrenna A. Accuracy of computer-aided oral implant surgery: a clinical and radiographic study. Int J Oral Maxillofac Implants. 2009;24(2):234-42.

16. Arisan V, Karabuda Z, Ozdemir T. Accuracy of Two Stereolithographic Guide Systems for Computer-Aided Implant Placement: A Computed Tomography-Based Clinical Comparative Study. J Periodontol. 2010 Jan 1;81:43-51.

17. Nikzad S, Azari A. A Novel Stereolithographic Surgical Guide Template for Planning Treatment Involving a Mandibular Dental Implant. J Oral Maxillofac Surg. 2008;66(7):1446-54.

18. Holst S, Blatz M, Eitner S. Precision for Computer-Guided Implant Placement: Using 3D Planning Software and Fixed Intraoral Reference Points. J Oral Maxillofac Surg. 2007 Apr 1;65:393-9.

19. Kola MZ, Shah AH, Khalil HS, Rabah AM, Harby NMH, Sabra SA, et al. Surgical templates for dental implant positioning; current knowledge and clinical perspectives. Niger J Surg Off Publ Niger Surg Res Soc. 2015;21(1):1-5.

20. Oh W-S, Saglik B. Use of a thermoplastic vacuum-formed matrix for secure engagement of an implant surgical template. J Prosthet Dent. 2008 Nov 1;100:326-7.

21. Geng W, Liu C, Su Y, Li J, Zhou Y. Accuracy of different types of computer-aided design/computer-aided manufacturing surgical guides for dental implant placement. Int J Clin Exp Med. 2015;8(6):8442-9. 
